Original Research Paper

\title{
Edge Preserved Image Compression Using Extended Shearlet Transform
}

\author{
${ }^{1}$ Thayammal, S. and ${ }^{2} \mathrm{D}$. Selvathi \\ ${ }^{1}$ Department of ECE Dhanalakshmi College of Engineering, Chennai-601 301, Tamil Nadu, India \\ ${ }^{2}$ Department of ECE Mepco Schlenk Engineering, College, Sivakasi 625 005, Tamil Nadu, India
}

\author{
Article history \\ Received: 30-01-2014 \\ Revised: $15-05-2014$ \\ Accepted: 04-08-2014 \\ Corresponding Author: \\ Thayammal, S. \\ Department of ECE \\ Dhanalakshmi College of \\ Engineering, Chennai-601 301, \\ Tamil Nadu, India \\ Email: tha_supa2003@yahoo.co.in
}

\section{Introduction}

In this rapidly changing world, a huge amount of online information is used either graphical or pictorial in nature.

Image compression is one of the key components to compress the data for specified channel bandwidths or storage requirements maintaining the highest possible quality. Image compression is the process of reducing the number of bits required to represent an image. Hence, the amount of memory required to store the data set is reduced (Gelli and Poggi, 1999; Mallat, 1989). It also reduces the amount of time required to transmit a data set over a communication link at a given rate.

\begin{abstract}
The motivation of the proposed compression method is to reduce the bit rate for image transmission or memory requirement for image storage while maintaining image quality. The edges are one of the prominent features in an image and they are essential for maintaining image quality. JPEG compression standards like JPEG98 and JPEG2000 produce visual artifacts in reconstructed image at low bit rate because, they didn't tailored about the detailed information like edges. Hence second generation coding introduced to preserve edge information, in which approximation and detailed information are separately encoded, so that, it introduces additional computational time and complexity. A multi directional anisotropic shearlet transform provides an optimally efficient representation of images with edges whereas wavelet transform have limited capability in dealing with edge information in all directions. Here, multidirectional transform called extended shearlet transform is used to uncorrelate the input gray level values with edge preserving capabiltiy. Hard thresholding method is applied to transform Partitionts and finally threshold output is encoded using Set analysis is performed between Edge Preserved Wavelet Transform coding (EPWT) and the extended shearlet transform coding. Image quality is measured objectively using peak signal-to-noise ratio, Structural Similarity Index (SSIM) and subjectively, using perceived image quality. The simulation results show that, the extended shearlet based compression technique is more efficient than EPWT coding Q Quantitative analysis on standard test images show that the proposed technique outperforms the EPWT coding technique by 0.16 to $1.46 \mathrm{~dB}$
\end{abstract} Keywords: Extended Shearlet Transform, Thresholding, Anisotropic Property
Generally the image comprises of low frequency and high frequency components. The low frequency components imply approximated information and high frequency components imply detailed information like edge information. There are many algorithms have been developed for image compression. But in general, transform coding method is preferred due to its ease of implementation. In order to achieve high compression ratio, most of the transform coding techniques use only low frequency components for compression. But in Human Visual system, selective neural cells have, undoubtedly, substantiated the perceptual importance of edges in an image (Marr and Ullman, 1981; Leventhal et al., 1998). 
JPEG was developed in 1992, using the DCT is simple and it is the widely used technique for compression. The input image is divided into blocks of $8 \times 8$ matrix. The DCT is applied to each block independently. Most of the DCT coefficients in each block are near zero values, which are quantized to zero and remaining coefficients are represented using reduced precision values that is determined by quantization scale. But, DCT based compression produces blocking artifactsfor high compression ratio.

In wavelet based compression (Al-Sammraie, 2011; Kousalyadevi and Ramakrishnan, 2012; Sridevi and Vijayakumar, 2013), the decomposition process splits the input image into one approximated and three detailed sub-images. The compression is made only on approximated image whereas the detailed sub-images are left to free. Hence, this wavelet transform also fails to preserve edge information in all direction. It is well known about DCT and DWT based compression algorithm produce visual artifacts at low bit rates. These artifacts are generally observed along high contrast regions such as object edges, which could severelydistort structural information and thus, impairing visual cognitive tasks.

Hence in recent years, there is improving work on image compression that attempt to preserve edge information. However wavelet fails to represent edge information in all direction. Now multidirectional transform is introduced to preserve edges in all directions. So many directional transforms have been proposed to overcome the limitations of traditional wavelets. The directional transforms are curvelets (Candes and Donoho, 2004), contourlets (Do and Vetterli, 2005) and bandelets (Peyre and Mallat, 2005). The construction of curvelets can't be built directly in the discrete domain and they do not provide a multiresolution representation of the geometry. Contourlets have less clear directional features than curvelets, which leads to artifacts in compression. Bandelets basis algorithm requires extensive computation.

In this proposed work, the image compression is based on anisotrpic extended shearlet transform. The extended shearlet transform is an affine system i.e., it preserves edge information while decomposition of an image. The shearlet transform is an efficient tool for representing structure information about the images (Yi et al., 2009; Krommweh, 2010). Adaptive the sholding of extended shearlet transform coefficients are determined and Set Partioning In Hierarchical Tree (SPIHT) coding technique is used to obatain compression. The proposed method is compared to EPWT coding method using CR, SSIM index and PSNR measures. In EPWT coding method, the wavelet coefficients and edge information are separately encoded. In the proposed method, the transform itself preserves edge information. Hence the proposed compression method is more efficient than EPWT coding method for a wide range of geometrical features for high coompression ratio, better image quality with less computational time.

The paper organized as follows. Section 2 provides the existing EPWT coding method. In section 3, the proposed scheme is introduced and discussed. Simulation results performed with standard test images are reported in section 4 and discussion in section 5 . Finally conclusion is drawn in section 6 .

\section{Edge Preserved Wavelet Transform (EPWT) Coding}

Image compression is one of the most visible applications of wavelets. Wavelet transforms are known to have excellent energy compaction characteristics. A wavelet is a foundation for representing images in various degrees of resolution (Servetto et al., 1999; Saha, 2000). The 2-D image $\mathrm{f}(\mathrm{x}, \mathrm{y})$ with size NXN can be decomposed as Equation 1:

$f=f_{j, a}+f_{j, D H}+f_{j, D V}+f_{j, D D}$

where, $f_{j, a}$ is the approximated image at level $j$ with half of the input image. The remaining three components are detailed information at level $j$ in horizontal, vertical and diagonal directions respectively. Wavelet transform is just the representation of functions by a wavelet, which is a mathematical function, dividing the function into various frequency components matching the resolution. Wavelet transformation methodology has been used because of the disadvantages in Fourier Transformation (Chappelier et al., 2004). The schematic diagram of EPWT coding is as shown in Fig. 1. In the encoder side, the edge information is extracted from the input image by canny edge detector and mapping of edge pixels are performed.

This mapping technique is used to convert edge pixel coordinates into map coordinates. Also this mapping process reduces the length of coordinates by 2, i.e., NXN number of edge pixel coordinates into $\mathrm{N}$ number of elements. The wavelet transform is applied to an input image. The wavelet transform coefficients are soft thresholded and encoded using SPIHT algorithm. These encoded bit stream are transmitted or stored along with edge information. At the decoder side, encoded bit stream is decoded. Then, inverse wavelet transform is applied to reconstruct the original image. Here mapping coordinates are converted into pixel coordinates, which are used to preserve edge information in the reconstructed image. 


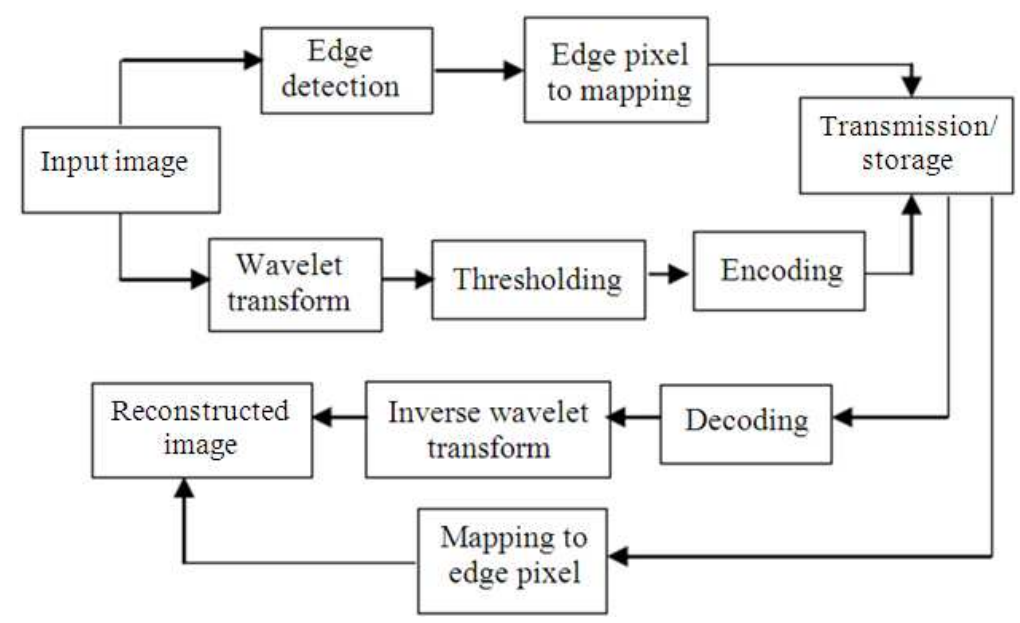

Fig. 1. Schematic diagram of image compression using EPWT coding method

\section{Image Compression Using Extended Shearlet Transform}

Discrete Shearlet Transform (DST) provides efficient multiscale directional representation. Our adaptive scheme using the DST significantly outperforms the wavelet transform. The shearlet decomposition functions orm a Parseval frame, but not a true basis. Recall that a Parseval frame for $L^{2}\left(R^{2}\right)$ is a collection of functions $\left\{\psi_{j}\right\}$ such that for any fin $L^{2}\left(R^{2}\right)$ Equation 2:

$$
\|f\|^{2}=\sum_{j}\left|<f, \psi_{j}>\right|^{2}
$$

Considering Parseval frames instead of bases is typically not a large disadvantage, since the only difference is that Parseval frames contain a certain amount of redundancy. Constructing shearlet bases is a non-trivial task, but it will be presented here nonetheless. First, let $\Psi$ be a "mother shearlet," which has certain technical properties (Lim, 2010; Lin and Smith, 2011).

Then the matrices $\mathrm{A}^{\mathrm{j}}$, which is the dilation matrix and $B^{l}$, which is the shear matrix are defined as Equation 3:

$$
A_{j}=\left(\begin{array}{cc}
2^{j} & 0 \\
0 & 2^{j / 2}
\end{array}\right) \text { and } B^{I}=\left(\begin{array}{ll}
1 & l \\
0 & 1
\end{array}\right)
$$

Now shearlet can be defined as:

$\psi_{j k l}=|\operatorname{det} A|^{j / 2} \psi\left(B^{l} A^{j} x-k\right)$

where, $j, l$ are in $\mathrm{Z}$ and $\mathrm{k}$ is in $Z^{2}$. With this definition, shearlets will be well-localized in both space and frequency domains. The shearlet $\Psi_{j k l}$ is supported in frequency domain on trapezoids at scale $2^{j}$, with orientation indexed by 1 and associated with spatial location indexed by the vector $\mathrm{k}$. Thus shearlets provide a decomposition of any $L^{2}\left(R^{2}\right)$ function into its frequency components according to the tiling of frequency domain by such trapezoids as shown in Fig. 2 .

The shearlet transform has the frame property. This will lead to a stable reconstruction of a given image. The shearlet frame is extended to produce a tight frame composed of directional orthonormal bases. Each of those directional orthonormal bases provides anisotropic window functions elongated along the direction determined by the action of shear matrix. This extended shearlet transform is basically same as that of shearlet transform except that extra basis elements to the shearlet are added to obtain an orthonormal basis for each shear matrix. The decomposition algorithm decomposes a given image into directional components using orthogonal transforms. The steps invovled in proposed extended shearlet based transform coding technique is as shown in Fig. 3. The algorithm steps are explained as follows.

Read an input image $f(x, y)$ with size NXN Equation 4:

$$
f(x, y)=0 \text { to } 255, \forall x, y \in Z \text {,i.e( }(1 \text { to } N)
$$

Extended Shearlet Transform is applied to an input image with three directions. It preserves edge information in the three directions with decorrelation of an input image Equation 5:

$$
F_{i}(m, n)=E S T\{f(x, y)\}, i=1 \text { to } 3
$$

where, $x, y, m n \in Z$ and $1 \leq x, y, m \& n \leq N$.

Adaptive thresholding of transformed coeffcients are obtained Equation 6:

$$
T_{A D A}=\text { selct_thresh }(F i) \text { and } F_{T}=T_{A D A}(F)
$$




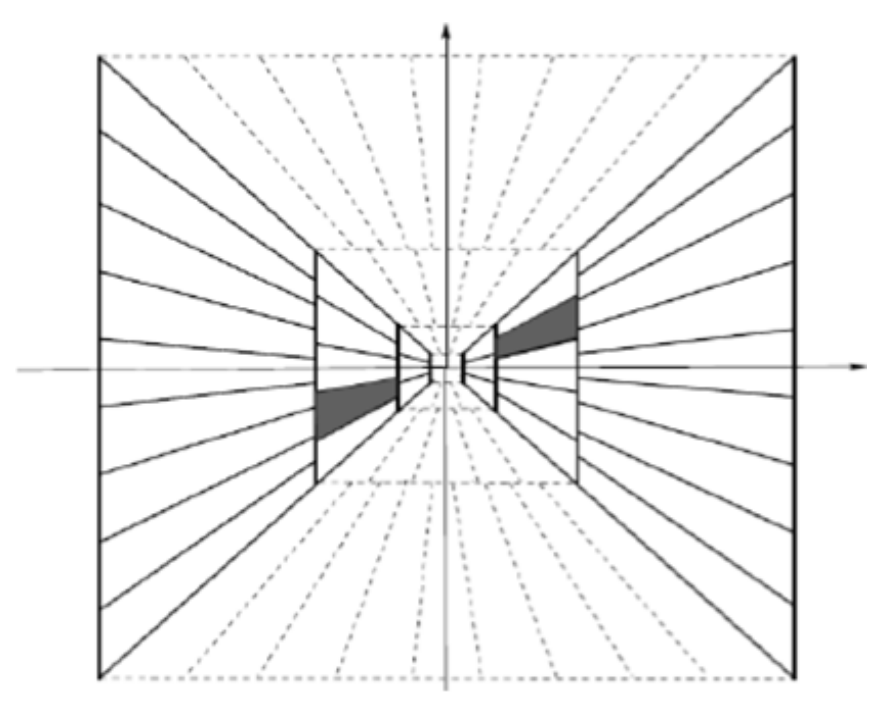

Fig. 2. Tiling of frequency domain induced by shearlets

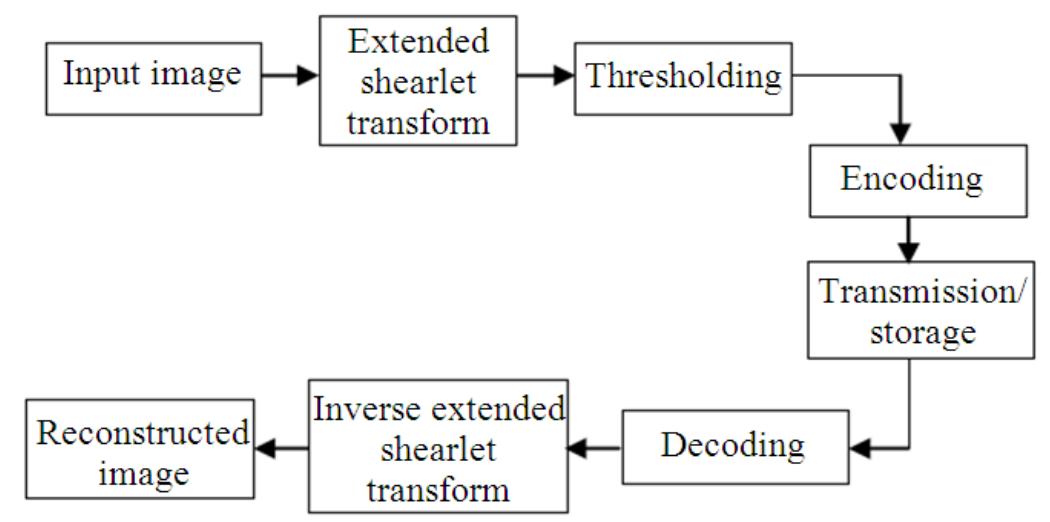

Fig. 3. Schematic diagram of image compression using extended shearlet transform coding method

Encoding of thresholded values is performed using Set Partitioning In Hierarchical Trees (SPIHT) technique Equation 7:

$$
F_{T(\text { comp })}=\operatorname{SPIHT}\left(F_{T}\right)
$$

SPIHT decoding of encoded bit stream is performed Equation 8:

$$
F_{(\text {decomp })}=S P I H T \_ \text {decode }\left(F_{T(\text { comp })}\right)
$$

The original image is reconstructed by applying Inverse Extended Shearlet Transform (IEST) to decoded information Equation 9:

$$
f_{a(\text { rec })}=\operatorname{IEST}\left(F_{(\text {decomp })}\right)
$$

The 2-D input image is efficiently decorrelated by extended shearlet transform. Thresholding is used to reduce the precision of the transform coefficients and also used to reduce the number of bits needed to represent the image. The output of threshold step is encoded by one of the wavelet coder like Set Partitioning In Hierarchical Tree (SPIHT). Then in output side, the SPIHT decoding id performed and inverse extended shearlet transform is applied to decoded value to get reconstructed image.

\section{Simulation Results}

The simulation results for both EPWT coding and extended shearlet transform coding are presented. For comparative analysis, standard images are taken like barbara, lena, goldhill, cameraman and rice. For performance analysis both objective and subjective measures are presented. Figure 4 shows performance comparison between EPWT and Extended Shearlet Transform coding method.

The reconstructed image using EPWT coding and extended shearlet transform coding is shown in Fig. $4 \mathrm{~b}$ and c respectively. From reconstrcuted images, it is observed that the extended shearlet transform coding preserves more edge information than EPWT coding.

The objective measures like compression ratio, PSNR SSIM and computational time are given in Table 1. 
Thayammal, S. and D. Selvathi / Journal of Computer Science 2015, 11 (1): 82.88 DOI: $10.3844 / j \operatorname{jssp} .2015 .82 .88$
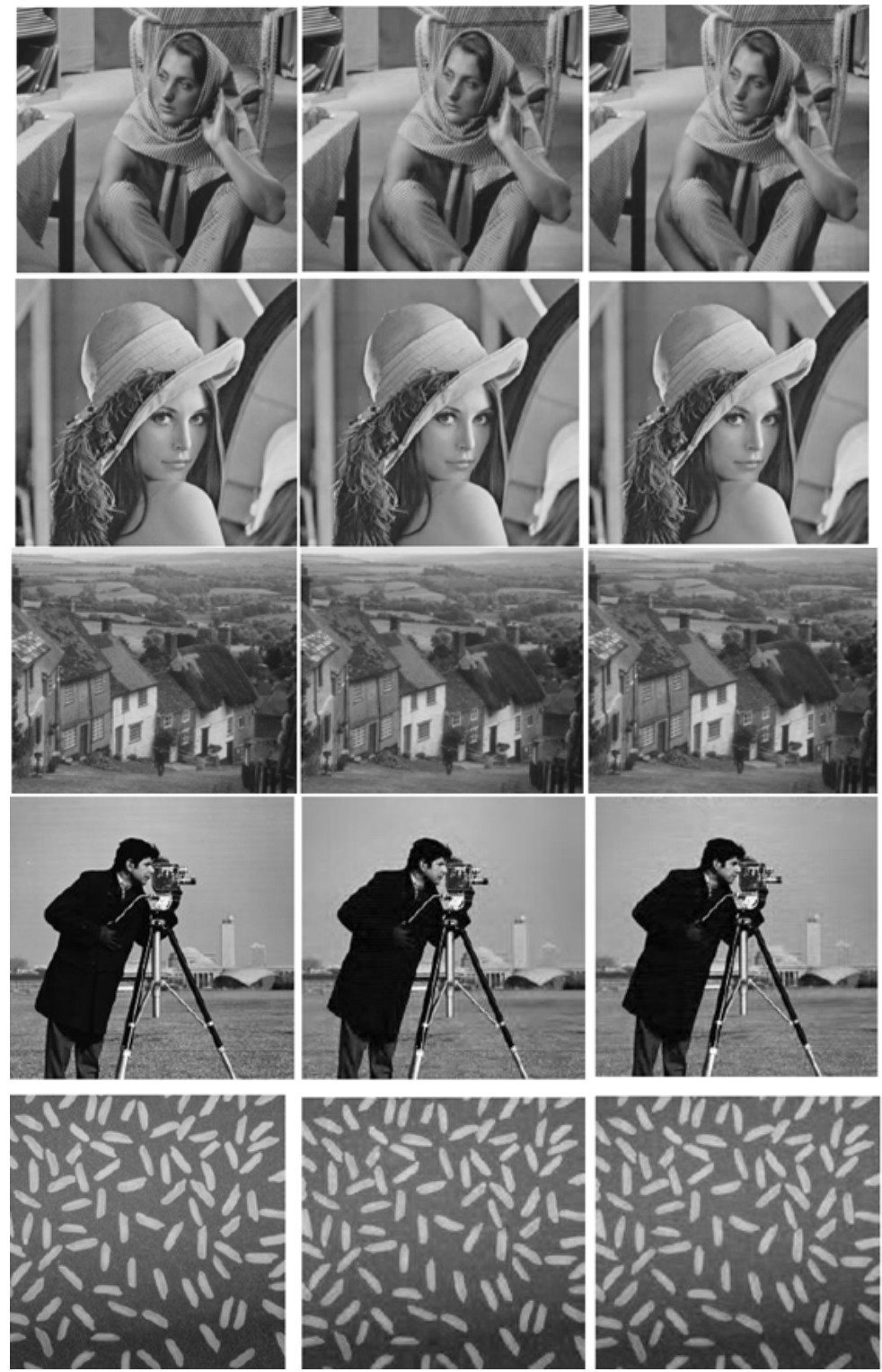

Fig. 4. Performance Comparison between EPWT and extended shearlet transform coding (a) Input image (b) Reconstructed image using EPWT and (c) Reconstructed image using extended shearlet transform coding

Table 1. Performance analysis of edge preserved wavelet basedcompression and extended shearlet based compression EPWT codingmethod Extended shearlettransform codingmethod

\begin{tabular}{lcccccccc} 
Input image & PSNR (dB) & CR & SSIM & $\begin{array}{c}\text { Computational } \\
\text { time (sec) }\end{array}$ & PSNR (dB) & CR & SSIM & $\begin{array}{l}\text { Computational } \\
\text { time(sec) }\end{array}$ \\
\hline Bar bara & 31.35 & 15.68 & 0.8938 & 2.80 & 32.78 & 17.20 & 0.9143 & 2.69 \\
Lena & 36.96 & 11.35 & 0.9345 & 2.57 & 37.12 & 11.30 & 0.9384 & 2.31 \\
Goldhill & 33.22 & 13.99 & 0.8690 & 2.69 & 33.79 & 14.06 & 0.8893 & 2.63 \\
Cameraman & 37.90 & 31.36 & 0.8678 & 1.26 & 32.00 & 28.60 & 0.8779 & 1.25 \\
Rice & 36.10 & 28.70 & 0.7916 & 1.29 & 30.45 & 29.40 & 0.8063 & 1.25 \\
\hline
\end{tabular}


This table shows that the extended shearlet transform coding produces higher PSNR as well as high CR with less computational time than EPWT coding. The important discussion is, this improved PSNR and CR using proposed extended shearlet transform coding is not achieved for all test images. It is observed that the cameraman and rice image produces low PSNR for the proposed method. But the subjective measure of perceived visual image quality is better than EPWT as shown in Fig. 4.

Though PSNR is the most widely used objective image quality metric, its values do not perfectly correlate with a perceived visual quality due to the nonlinear behavior of the human visual system. Hence one more important metrics Stuctural SIMlarity (SSIM), which is perfectly correlated with perceived visual quality is determined, for all test images. The SSIM index is a method for measuring the similarity between two images. SSIM is designed to improve on traditional methods like Peak Signal-to-Noise Ratio (PSNR) and Mean Squared Error (MSE), which have proven to be inconsistent with human eye perception Equation 10:

$\operatorname{SSIM}(x, y)=\frac{\left(2 \mu_{x} \mu_{y}+c_{1}\right)\left(2 \sigma_{x y}+c_{1}\right)}{\left(\mu_{x}^{2} \mu_{y}^{2}+c_{1}\right)\left(\sigma_{x}^{2}+\sigma_{y}^{2}+c_{2}\right)}$

$\mu_{x}=$ The average of $\mathrm{x}$ and $\mu_{y}$ the average of $y$

$\sigma_{\mathrm{x}}^{2}=$ The variance of $\mathrm{x}$ and $\sigma_{\mathrm{y}}^{2}$ the variance of $y$

$\sigma_{x y}=$ The covariance of $\mathrm{x}$ and $\mathrm{y}$

$c_{1}=\left(k_{1} L\right)^{2}, c_{2}=\left(k_{2} L\right)^{2}$ two variables to stabilize the division with weak denominator

$L=$ The dynamic range of the pixel-values (typically this is $2^{\mathrm{b}}-1, \mathrm{~b}$ number of bits/pixel)

$k_{1}=0.01$ and $k_{2}=0.03$

From Table 1, it observed that, the proposed method produces improved values of SSIM for all test images.

\section{Discussion}

In this study, the compression of an image using extended shearlet transform and EPWT are discussed. Compared to EPWT method, the proposed method produces high PSNR for barbara $(32.78 \mathrm{~dB})$, lena $(32.78$ $\mathrm{dB})$ and goldhill $(33.79 \mathrm{~dB})$ images for the improved average compression ratio is $1 \%$ with less computational time. But the proposed method produces low PSNR values for cameraman $(32 \mathrm{~dB})$ and rice $(30.45 \mathrm{~dB})$ images. But the perceived image quality is better in proposed method. This results because of the PSNR values are not correlated with perceived image quality. Hence another perfoamance measure called SSIM is taken for comparative analysis. The SSIM index is analysed for both the methods with all test images. The proposed method provides improved average SSIM index 0.1 at high compression ratio.

\section{Conclusion}

The two transform coding techniques, based on wavelet transform and shearlet transform are presented. In EPWT coding, edges are processed separately and transmitted/stored along with wavelet transform coding output. In shearlet based method, extended shearlet transform is used and which is obtained by applying shear transforms on the discrete domain in two directions followed by the anisotropic wavelet transforms. From simulation results, it observed that the extended shearlet transform coding improves compression performance by the factors of compression ratio, PSNR and computational time. The issue that need to resolve in this proposed method is the complexity is increased with increasing number of iterations and directions. Thus, the future study will focus on analysis in image compression of Landsat-7 images using this extended shearlet transform. In Landsat-7, out of 8 band, 7 band images are in spatial resolution of $60 \mathrm{~m}$ and one band with spatial resolution of $15 \mathrm{~m}$. Only a few iterations and directions of shear can still provide good approximations of an input image (Lim, 2010). So need to analyze the optimum number of iterations and directions for 60 and $15 \mathrm{~m}$ spatial resolution of Lantsat-7 multispectral images.

\section{Author's Contributions}

All authors equally contributed in this work.

\section{Ethics}

This article is original and contains unpublished material. The corresponding author confirms that all of the other authors have read and approved the manuscript and no ethical issues involved

\section{References}

Al-Sammraie, M.F.H., 2011. Colored image compression using gradient adjustment prediction based wavelet. J. Comput. Sci., 7: 304-313. DOI: $10.3844 /$ jessp.2011.304.313 
Candes, E.J. and D.L. Donoho, 2004. New tight frames of curvelets and optimal representations of objects with piecewise $\mathrm{C} 2$ singularities. Commun. Pure Applied Math., 57: 219-266.

DOI: $10.1002 /$ cpa.10116

Chappelier, V., C. Guillemot and S. Marinkovic, 2004. Image coding with iterated contourlet and wavelet transforms. Processing of the International Conference onImage Processing, Oct.24-27, IEEE Xplore Press, pp: 3157-3160. DOI: 10.1109/ICIP.2004.1421783

Do, M.N. and M. Vetterli, 2005. The contourlet transform: An efficient directional multiresolution image representation. IEEE Trans. Image Proc., 14: 2091-2106. DOI: 10.1109/TIP.2005.859376

Gelli, G. and G. Poggi, 1999. Compression of multispectral images by spectral classification and transform coding. IEEE Trans. Image Proc., 8: 476-489. DOI: $10.1109 / 83.753736$

Kousalyadevi, R. and S.S. Ramakrishnan, 2012. Performance analysis of multi spectral band image compression using discrete wavelet transform. J. Comput. Sci., 8: 789-795.

DOI: $10.3844 /$ jcssp.2012.789.795

Krommweh, J., 2010. Tetrolet transform: A new adaptive haar wavelet algorithm for sparse image representation. J. Vis. Comm. Image Represent., 21: 364-374. DOI: 10.1016/j.jvcir.2010.02.011

Leventhal, A.G., Y.C. Wang, M.T. Schmolesky and Y. Zhou, 1998. Neural correlates of boundary perception. Vis. Neurosci., 15: 1107-1118.

Lim, W.Q., 2010. The discrete shearlet transform: A new directional transform and compactly supported shearlet frames. IEEE Trans. Image Proc., 19: 1166-1180. DOI: 10.1109/TIP.2010.2041410
Lin, J. and M.J.T. Smith, 2011. Two-band hybrid FIRIIR filters for image compression. IEEE Trans. Image Proc., 20: 3063-3072. DOI: 10.1109/TIP.2011.2134860

Mallat, S.G., 1989. Multifrequency channel decompositions of images and wavelet models. IEEE Trans. Acoust. Speech Signal Proc., 37: 2091-2110. DOI: 10.1109/29.45554

Marr, D. and S. Ullman, 1981. Directional selectivity and its use in early visual processing. Proc. Roy. Soc. Lond., 208: 151-180. DOI: 10.1098/rspb.1981.0001

Peyre, G. and S. Mallat, 2005. Discrete Bandelets with geometric orthogonal filters. Proceedings of the IEEE International Conference on Image Processing, Sept. 11-14, IEEE Xplore Press, pp: 65-68. DOI: 10.1109/ICIP.2005.1529688

Saha, S., 2000. Image compression-from DCT to Wavelets: A review. ACM Cross Words Students Magazine, 6: 12-21. DOI: 10.1145/331624.331630

Servetto, S.D., K. Ramchandran and M.T. Orchard, 1999. Image coding based on a morphological representation of wavelet data. IEEE Trans. Image Proc., 8: 1161-1174. DOI: 10.1109/83.784429

Sridevi, S. and V.R. Vijayakumar, 2013. Medical image compression technique using listless set partitioning in hierarchical trees and contextual vector quantization for brain images. DOI: 10.3844/jcssp.2013.1181.1189

Yi, S., D. Labate, G.R. Easley and H. Krim, 2009. A shearlet approach to edge analysis and detection. IEEE Trans. Image Proc., 18: 929-941. DOI:10.1109/TIP.2009.2013082 